\title{
SISTEMA DE DETECÇÃO AUTOMÁTICA DE PAROXISMOS EPILEPTOGÊNICOS EM SINAIS DE ELETROENCEFALOGRAMA
}

\author{
Fernanda Isabel Marques Argoud \\ fargoudeieb.ufsc.br
}

Fernando Mendes de Azevedo

azevedo@ieb.ufsc.br

\author{
José Marino Neto \\ marino@ccb.ufsc.br
}

Instituto de Engenharia Biomédica, Departamento de Engenharia Elétrica

Universidade Federal de Santa Catarina, Florianópolis, SC, Brasil, 88040-900

\begin{abstract}
This paper presents an automatic computational system to detect and classify epileptogenic transients in electroencephalogram (EEG), to aid Epilepsy diagnosis. Due to great variability on the morphology of these events, conventional tools of pattern recognition are not able to distinguish between normal and epileptogenic activity, and visual detection is a very time-consuming task. So, tools and methods normaly used to detect these events try to imitate human expertise. False positive detection represents considerable impediment to extensive use of automatic systems by EEG readers. The proposed system applies Wavelet Transform to extract only epileptogenic features from the EEG signals, and a group of specialized Artificial Neural Networks (ANNs) to distinguish spike and sharp wave events from normal background activity. Two ANNs are used cooperatively, allowing greater flexibility in adjusting system's sensibility and specificity, to improve performance. When sensibility and specifity are set to be equal, system's performance achieves $80 \%$.
\end{abstract}

Artigo Submetido em 19/08/03

1a. Revisão em 06/10/03;

2a. Revisão em 16/02/04;

Aceito sob recomendação do Editor Associado

Prof. Dr. José Roberto Castilho Piqueira
KEYWORDS: Automatic Detection, Epilepsy, Neural Networks, Spike, Wavelet Transform.

\section{RESUMO}

Este artigo apresenta uma proposta de sistema computacional automático para detecção e classificação de transientes epileptogênicos em eletroencefalograma, visando auxiliar no diagnóstico de Epilepsia. Devido à grande variabilidade das morfologias destes eventos, ferramentas convencionais de reconhecimento de padrões não são capazes de distinguir entre a atividade normal e a epileptogênica, e a análise visual é uma tarefa bastante demorada. Então, as ferramentas e métodos normalmente utilizados para detectar estes eventos tentam imitar o procedimento e o raciocínio de especialistas humanos. As detecções falso-positivas representam um grande impedimento para o uso extensivo de sistemas automáticos, por parte de eletroencefalografistas. O sistema proposto aplica Transformada Wavelet para extrair apenas as características epileptogênicas dos sinais de EEG, e um grupo de Redes Neurais Artificiais (RNAs) para distinguir entre eventos tipo espícula e onda aguda da atividade normal de fundo. Duas RNAs são usadas em conjunto, permitindo maior flexibilidade no ajuste da sensibilidade e da especificidade do sistema, como forma de aumentar o desempenho deste. Quando os valores de sensibilidade e 
especificidade são igualados, o desempenho do sistema atinge $80 \%$.

PALAVRAS-CHAVE: Detecção Automática, Epilepsia, Espícula, Redes Neurais, Transformada Wavelet.

\section{INTRODUÇÃO}

\subsection{Motivação}

No diagnóstico da doença Epilepsia utiliza-se amplamente a análise visual de sinais de eletroencefalograma, no qual podem ser encontrados eventos que são fortemente relacionados a foco epiléptico. Tais eventos são também conhecidos por padrões epileptogênicos, em oposição àqueles sinais cuja morfologia é semelhante às destes paroxismos, mas que não estão relacionados à doença, e que são chamados de padrões epileptiformes.

Os eventos epileptogênicos mais freqüentes em pacientes epilépticos e significativos para o diagnóstico são as espículas e as ondas agudas (Figura 1). Na realidade, a diferença entre estes paroxismos deve-se principalmente ao tempo de duração do evento, que é maior nas ondas agudas que nas espículas.
Apesar destes padrões serem facilmente identificáveis pelos profissionais treinados na leitura de sinais de EEG, os eletroencefalografistas ou "EEGers", a automatização desta detecção não foi conseguida até hoje, de fato. Isto ocorre principalmente porque os sistemas de detecção automática de eventos epileptogênicos desenvolvidos até o momento tendem a erroneamente identificar como positivos, um número muito grande de eventos epileptiformes. Dentre os eventos epileptiformes que mais interferem e prejudicam a detecção automática de eventos epileptogênicos pode-se destacar o ruído de alta freqüência, os movimentos oculares e piscadas, e a atividade alfa (Figura 2), principalmente por serem muito freqüentes em sinais de EEG e por apresentarem morfologia bastante semelhante à das espículas e ondas agudas. Também, a baixa especificidade dos sistemas ocorre porque os sinais de EEG variam de paciente para paciente e variam, no mesmo paciente, sob diferentes estados de consciência e comportamentais. Assim, fica difícil estabelecer-se um modelo computacional adequado de paroxismo, para que seja possível diferenciálo das demais atividades presentes no EEG.

É importante lembrar que a análise visual é um processo bastante lento e cansativo, já que o EEGer deve revisar atentamente dezenas, às vezes centenas (dependendo da duração total do exame), de páginas impressas com 10 a 30 s de EEG, organizados em 24 a 32 canais cada, distribuídos
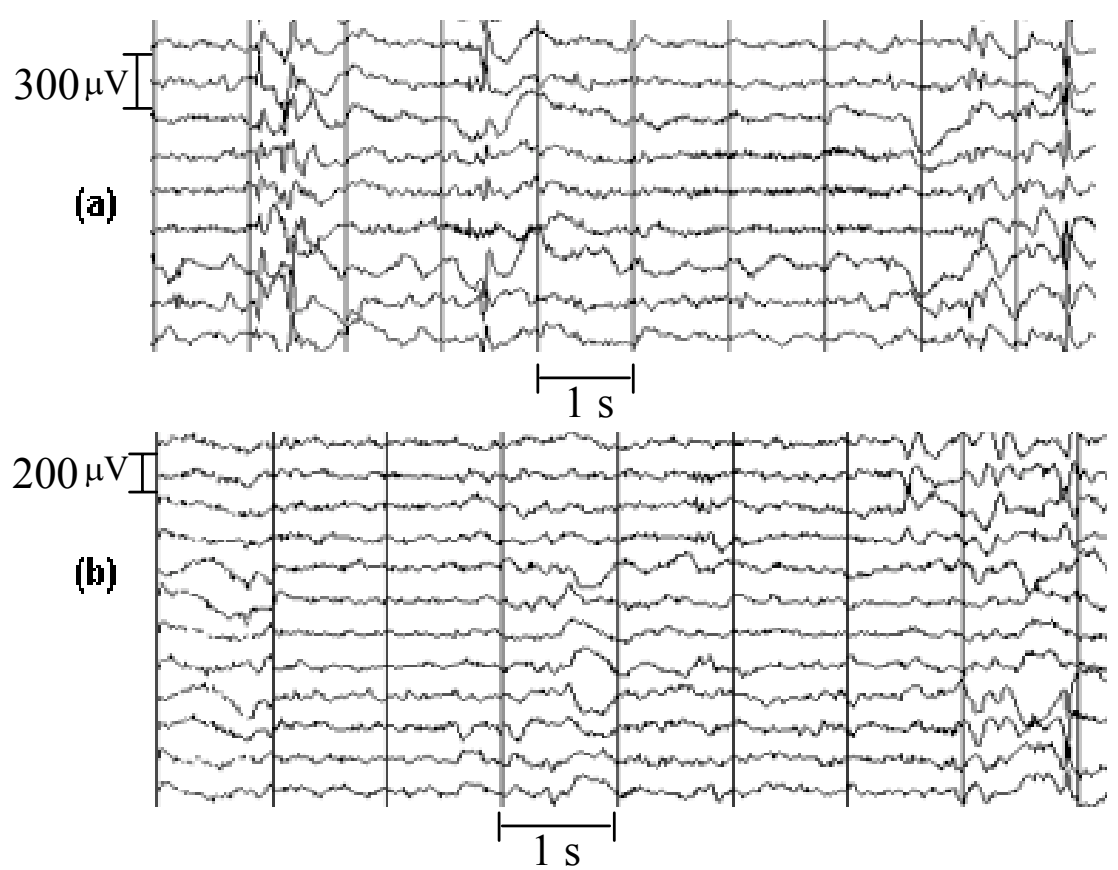

Figura 1 - Em (a): espículas rápidas (menos de $70 \mathrm{~ms}$ de duração) destacando-se visualmente da atividade de fundo do EEG na segunda, quarta e décima colunas de tempo. Em (b): ondas agudas (com até $200 \mathrm{~ms}$ de duração) na sétima e oitava colunas. 
ao longo de todo o córtex cerebral, na busca por alguns padrões de sinal que têm durações de apenas 70 a $200 \mathrm{~ms}$. Daí a maior necessidade de se automatizar o processo de detecção e classificação de eventos epileptogênicos. No caso dos registros de EEG de longa duração, nos quais aumenta a probabilidade de se encontrar espículas caso o paciente seja epiléptico, a especificidade ${ }^{1}$ dos sistemas torna-se mais importante que a sensibilidade ${ }^{2}$. Apesar disto, grande parte dos sistemas automáticos propostos até o presente não conseguem atingir taxas de sensibilidade e especificidade superiores a $50 \%$, ou seja, acima da aleatoriedade (Wilson e Emerson, 2002).

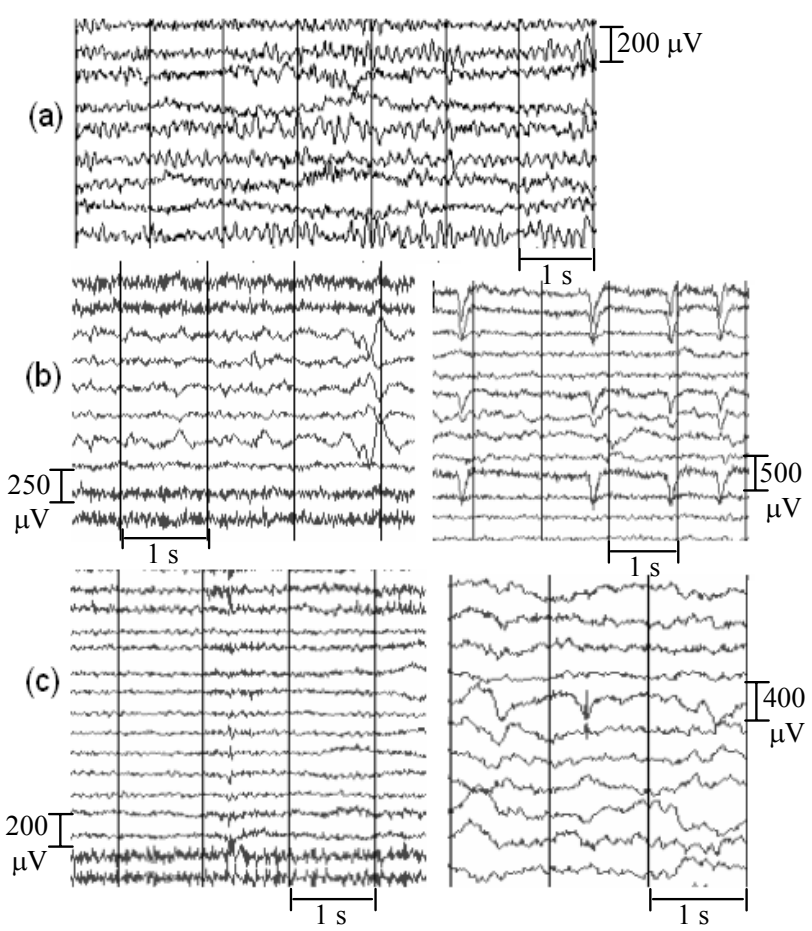

Figura 2 - (a) Padrões epileptiformes devidos a ritmo alfa. (b) Padrões epileptiformes devidos a movimentos oculares (à esquerda) e devidos a piscadas (à direita). (c) Padrões epileptiformes de alta freqüencia devidos a artefatos de paciente e eletrodo.

Enfim, dada a dificuldade em se estabelecer um modelo matemático formal para os padrões epileptogênicos, optou-

\footnotetext{
${ }^{1}$ Especificidade ou Seletividade em um processo de detecção de padrões refere-se à habilidade da ferramenta em discriminar padrões relevantes dos irrelevantes, ou seja, é inversamente proporcional à freqüência de deteç̧ões falsamente-positivas apresentada pela ferramenta. É dada por $(100 \times \mathrm{VN}) /(\mathrm{VN}+\mathrm{FP})$, sendo $\mathrm{VN}$ o número de verdadeiros negativos e $\mathrm{FP}$, o número de detecções falsamente positivas (Gabor e Seyal, 1992).

2 Sensibilidade em um processo de detecção de padrões refere-se à habilidade da ferramenta em identificar os padrões relevantes, ou seja, é inversamente proporcional à freqüência de detecções falsamente-negativas apresentada pela ferramenta. É dada por $(100 \times \mathrm{VP}) /(\mathrm{VP}+\mathrm{FN})$, sendo VP o número de detecções verdadeiramente positivas e $\mathrm{FN}$, o número de deteç̧ões falso negativas (padrão relevante erroneamente não-detectado pela ferramenta) (Gabor e Seyal, 1992).
}

se por utilizar ferramentas de Inteligência Artificial (IA), que permitam trabalhar-se com identificação de padrões quando não se tem uma definição precisa dos parâmetros a serem analisados.

Também a Transformada Wavelet está sendo utilizada, por permitir representar sinais com resolução em tempo e em freqüência simultaneamente, por poder ressaltar detalhes de interesse em sinais particulares, por dispor de um algoritmo rápido de transformação, etc.

\subsection{Estado da Arte}

Várias metodologias e algoritmos foram propostos desde 1976, com a publicação das primeiras abordagens de Gotman e Gloor (1976), na tentativa de se automatizar a detecção de espículas, e outros eventos epileptogênicos, em sinais de EEG.

Uma revisão recente sobre o estado da arte foi realizada por Wilson e Emerson (2002). Nesta, os autores lembram que a importância da detecção automática de espículas vai além do diagnóstico de epilepsia, mas também é fundamental para o tratamento e investigação de indicação cirúrgica, por exemplo.

De modo geral, pode-se classificar os trabalhos mais conhecidos até o presente em quatro classes básicas, segundo a metodologia que empregam: os paramétricos, os de processamento de sinais, os que utilizam inteligência artificial e os híbridos.

Os métodos paramétricos procuram estabelecer um modelo matemático para as espículas. Para tanto, fazem levantamentos estatísticos das características supostamente relevantes e particulares na morfologia dos eventos espiculares, e treinam sistemas para identificar estas características nos sinais. Nesta categoria pode-se destacar o trabalho de Gotman e Wang (1991), que apesar de não ser recente, é utilizado até hoje como base para sistemas paramétricos, e Pereira (2003), que desenvolveu e propôs uma série de novos parâmetros de modelagem de espículas.

Os trabalhos baseados em técnicas de processamento digital de sinais fundamentalmente utilizam ferramentas matemáticas clássicas para separar os eventos de interesse (espículas, ondas agudas, crises eletrográficas, etc), das demais atividades no sinal de EEG. Esta extração de características normalmente é realizada através de filtragens e transformações digitais. Destacam-se os trabalhos de Stelle e Comley (1989 e 1990), que usaram Transformada de Hilbert e Distribuição de Wigner, e Attelis et alii (1997), que utilizaram Transformada Wavelet Biortogonal.

Os sistemas aqui ditos como os que utilizam técnicas de Inteligência Artificial (IA) freqüentemente incluem também outras técnicas, mas foram considerados em separado 
porque se baseiam na tentativa de se imitar o discernimento dos EEGers, na tarefa de detecção de eventos epileptogênicos. Isto é, empregam redes neurais, informações de contexto em regras de produção e/ou raciocínio baseado em casos para modelar a capacidade humana de reconhecimento de padrões e de reutilização de conhecimento na inferência sobre problemas presentes. $\mathrm{Ou}$ seja, não há um estabelecimento formal de modelos "definitivos" para espículas e tampouco o enfoque é dado no processamento dos sinais. Dentro desta categoria de sistema destaca-se o trabalho de Pradhan et alii (1996), que utilizaram uma rede do tipo Vetor de Quantização Linear (LVQ) para analisar os sinais de EEG.

Já os sistemas híbridos são definidos aqui como aqueles que se utilizam com igual relevância de mais de um tipo das abordagens supracitadas. A maioria dos sistemas bem sucedidos enquadra-se nesta categoria. Pode-se destacar os trabalhos de: Gabor e Seyal (1992), Webber et alii (1994), Feucht et alii (apud Wilson e Emerson, 2002) e Wilson et alii (1999), os quais usaram sinais parametrizados como entradas para redes neurais artificiais; de Kalayci e Özdamar (1995), que abordaram o uso da Transformada Wavelet como ferramenta de pré-processamento do EEG para treinamento de redes neurais; e de Hoffmann et alii (1996) que usaram a Transformada de Hilbert para préprocessar os sinais a serem apresentados a redes neurais. Destacam-se especialmente os trabalhos de Dümpelmann e Elger (1999), que compararam a detecção manual de espículas a várias abordagens automáticas, em sinais de EEG intracranianos, e o trabalho de Pereira (2003), que investigou e comparou as várias formas de préprocessamento para aplicação de sinais de EEG a redes neurais artificiais.

Apesar de inúmeros outros trabalhos terem sido publicados recentemente, os autores escolheram mencionar aqui preferencialmente aqueles que reportaram boas taxas de desempenho, obtidas em pelo menos 60 minutos de registros variados, de diferentes pacientes, e sem preparação prévia dos dados (além daquelas do próprio sistema), que pudessem aumentar artificialmente as taxas de desempenho.

\subsection{Objetivos}

Assim, o objetivo deste trabalho foi o de investigar o uso de ferramentas matemáticas de IA e Transformada Wavelet na detecção e classificação automática de eventos epileptogênicos em sinais de eletroencefalograma, com vistas ao desenvolvimento de um sistema que apresente bom desempenho, simultaneamente em sensibilidade e especificidade.

\section{MATERIAIS E MÉTODOS}

\subsection{A Transformada Wavelet na detecção automática de espículas}

A Transformada Wavelet tem sido amplamente utilizada no reconhecimento automático de eventos epileptogênicos por ser adequada ao tratamento de sinais estocásticos, que contenham grande número de transientes, por permitir uma representação tempo-freqüência com boas resoluções simultâneas nos dois domínios, por permitir grande compactação de dados quando houver escolha adequada da função wavelet de decomposição e por dispor de um algoritmo rápido, de implementação simples (Argoud, 2001).

O algoritmo Fast Wavelet Transform (FWT) implementa a decomposição de um sinal discreto $\mathbf{x ( t )}$ através do "Esquema de Lifting", proposto por Sweldens (1996), para filtragem digital utilizando bancos de filtros espelho multiresolução em quadratura. Ou seja, um conjunto de filtros passa-baixas $\mathbf{H}(\mathbf{t})$ vai extrair as características de média (ou "aproximações") do sinal, enquanto um conjunto de filtros passa-faixas $\mathbf{G ( t )}$ extrairá as características de frequências não-nulas (ou "detalhes") do sinal x(t) (Figura 3). Ambos os filtros ortogonais $\mathbf{H}(\mathbf{t})$ e $\mathbf{G}(\mathbf{t})$ são derivados da função wavelet-mãe básica (Argoud, 2001).

O maior problema na utilização da Transformada Wavelet é a determinação da melhor wavelet-mãe, a ser utilizada para um caso particular de sinal. No caso específico do problema de aplicação a sinais de EEG, com vistas à detecção de atividade epileptogênica, normalmente esta escolha é arbitrária ou empírica (Kalayci e Özdamar, 1995; Dümpelmann e Elger, 1999; Schiff et alii, 1994; Khan e

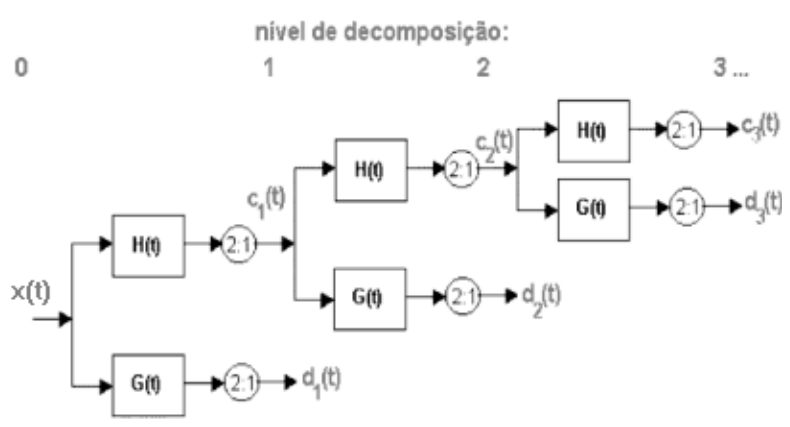

Figura 3 - Esquema de Lifting de Sweldens (1996). Os filtros $G(t)$ extraem os detalhes $d_{i}(t)$, do sinal $x(t)$. Os filtros $H(t)$ extraem as aproximações, em níveis cada vez menores de refinamento (diminuição da banda de freqüências), com aumento do nível de decomposição $i$.

Após cada etapa de decomposição é feita uma decimação do sinal (Argoud, 2001). 

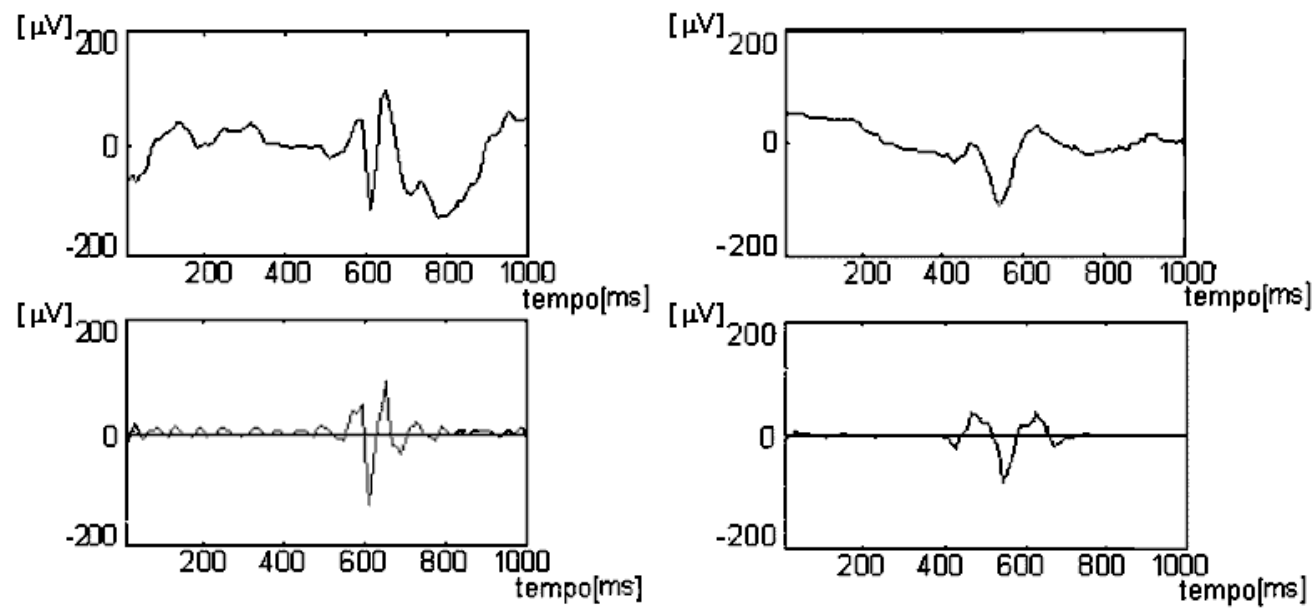

Figura 4 - À esquerda: acima, trecho de $1 \mathrm{~s}$ de sinal de EEG contendo espícula; em baixo, sinal reconstruído a partir do detalhe 2 da Coiflet 1. À direita: acima, trecho de $1 \mathrm{~s}$ de sinal de EEG contendo onda aguda; em baixo, sinal reconstruído a partir do detalhe 3 da Coiflet 1.

Gotman, 2003). Não obstante, esta escolha é crítica para a obtenção de resultados satisfatórios com o uso da Transformada Wavelet para detecção de padrões, mais especificamente de espículas (Argoud et alii, 2003).

Um estudo recente dos autores (Argoud et alii, 2003) constatou que quando o objetivo é utilizar-se sinais de EEG reconstruídos após decomposição por FWT, de modo a ressaltar os eventos epileptogênicos com relação à atividade de fundo, a função wavelet Coiflet $1^{3}$, nos níveis de decomposição 2 e 3 é, dentre as mais conhecidas, aquela que melhor identifica eventos epileptogênicos em meio à atividade de fundo normal do EEG, facilitando o processo de deteç̧ão (Argoud et alii, 2003). Sobretudo, o artigo comprova que várias funções wavelet, tais como as Biortogonais 1.1, 1.3, 1.5 e 6.8, Daubechies 1, 6, 9 e 19 e Symlet 6, nas condições citadas são inadequadas à esta tarefa, por não se aproximarem matematicamente das espículas e ondas agudas (Argoud et alii, 2003).

Já os níveis de decomposição foram escolhidos por terem sido aqueles que concentraram as maiores energias para coeficientes devidos aos eventos epileptogênicos. Em outras palavras, para sinais adquiridos a $100 \mathrm{~Hz}$ e préfiltrados em $0,5-40 \mathrm{~Hz}$ (procedimento normalmente adotado para aumento da relação sinal/ruído), a decomposição por Coiflet1 - nível de decomposição 2 representa uma filtragem na faixa aproximada de $12,5-23 \mathrm{~Hz}$, enquanto a decomposição Coiflet1 - nível de decomposição 3 filtra o sinal na banda $6,25-12,5 \mathrm{~Hz}$, aproximadamente, o que coincide com a banda de interesse para eventos

\footnotetext{
${ }^{3}$ A função wavelet Coiflet 1 é ortogonal, tem regularidade pobre mas está próxima da simetria, possui suporte compacto e filtros FIR e suas funções de escala e wavelet apresentam momentos de decaimento (Argoud, 2001; Argoud et. alii, 2003; Sheng, 1996)
}

epileptogênicos, em sinais de EEG, que é de 2 a $20 \mathrm{~Hz}$. O nível de decomposição 1 (22,0-40 Hz, aproximadamente) também foi capaz de detectar as espículas, principalmente aquelas mais rápidas, mas como foi também mais susceptível aos eventos ruidosos, foi descartada. Já os níveis de decomposição superiores a 3 (filtragens em bandas inferiores a $6,0 \mathrm{~Hz}$ ) não foram capazes de detectar as espículas e ondas agudas, tendo sido também descartadas.

Ao longo do trabalho, ficou evidente que os coeficientes d2 eram mais adequados à detecção de espículas, enquanto os coeficientes $\mathrm{d} 3$, à detecção de ondas agudas. Com alguma vantagem para o nível de decomposição 2, por ter sido mais localizado no tempo e menos susceptível a movimentos oculares, presentes no EEG. Por outro lado, este nível foi mais sensível à atividade muscular ruidosa, de alta freqüência.

Assim, os vetores de detalhe reconstituídos a partir do nível de decomposição 2 (coeficientes d2) foram utilizados para ressaltar as espículas e, os detalhes do nível 3 (coeficientes d3), para ressaltar as ondas agudas, com relação aos demais eventos presentes nos sinais de EEG (Figura 4). É interessante salientar que o ritmo alfa foi consideravelmente filtrado pela Coiflet 1 .

\subsection{O uso de Redes Neurais na detecção automática de espículas}

A Transformada Wavelet é capaz de ressaltar os eventos epileptogênicos com relação às demais atividades, amplificando-os. Porém não é suficiente para o reconhecimento automático devido à grande variabilidade destes eventos. Ou seja, eventualmente a FWT acaba 


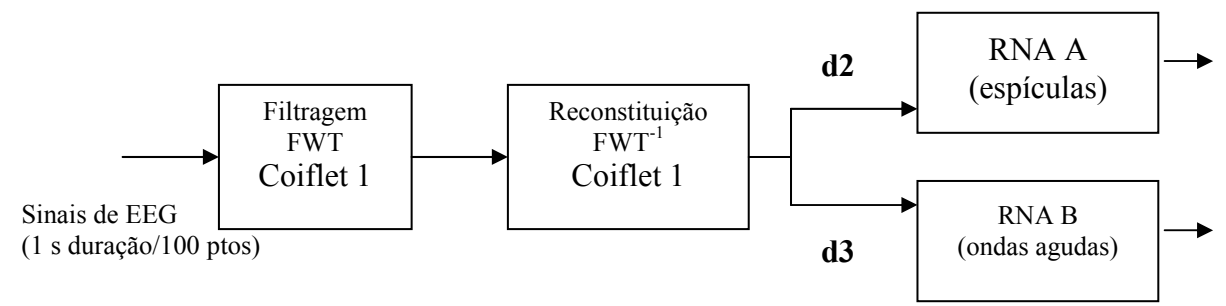

Figura 5 - Esquema simplificado do sistema de reconhecimento automático de eventos epileptogênicos em EEG. Os trechos de sinais de EEG com 100 pontos são filtrados e reconstituídos por Transformada Wavelet, Coiflet 1 - nível de decomposição 3. Posteriormente, os sinais de detalhe 2 e 3 já condicionados são submetidos a um banco de RNAs, para a detecção propriamente dita.

amplificando também eventos epileptiformes, o que acaba aumentando a taxa de detecções falso-positivas.

Para minimizar este problema, optou-se por treinar duas Redes Neurais Artificiais (RNAs) para o reconhecimento propriamente dito dos padrões epileptogênicos. Mais especificamente, uma rede foi treinada para reconhecer espículas (Rede A) e outra para reconhecer ondas agudas (Rede B), tal como ilustrado na Figura 5.

As RNAs são sistemas matemáticos complexos, do paradigma conexionista de Inteligência Artificial, constituídos de elementos representando algumas das características dos neurônios biológicos, os quais encontram-se interligados entre si através de conexões ponderadas, por pesos sinápticos (De Azevedo, 1997).

O uso das RNAs tornou-se popular por estas permitirem análises complexas, não-lineares (como em reconhecimento de padrões), de uma forma relativamente simples, com bom desempenho e à revelia da ausência de justificativas matemáticas e considerações mais apuradas, acerca das estruturas e parâmetros de redes escolhidos para cada aplicação.

No caso deste trabalho, optou-se pelo uso das redes clássicas, do tipo Perceptron Multicamadas (MLP), treinadas com algoritmo Backpropagation, pela facilidade de implementação, pelo desempenho que costumam apresentar e pela disponibilidade de bibliografia farta (De Azevedo, 1997).

As redes implementadas que obtiveram melhores resultados apresentam topologia de 4 camadas, sendo uma de entrada com 100 neurônios (em função do número de pontos, nos vetores de 100 patamares de amplitudes), 21 neurônios na segunda camada, 4 neurônios na terceira camada e apenas um neurônio na camada de saída. Os números de neurônios nas camadas ocultas intermediárias foram estimados, segundo um critério empírico, como médias quadráticas entre os tamanhos da camada de entrada e da camada de saída. As funções de saída dos neurônios das duas camadas intermediárias são do tipo tangente hiperbólica (bipolar), e a função de saída na camada de saída é do tipo função logística (unipolar).

$\mathrm{O}$ conjunto de treinamento da rede $\mathrm{A}$, de reconhecimento de espículas, foi constituído de 99 trechos de $1 \mathrm{~s}$, de 9 registros de EEG provenientes de 7 pacientes, comprovadamente epilépticos. Os sinais foram transformados e, em seguida, reconstruídos a partir do nível de decomposição 2, Wavelet de Coiflet1. Destes 99 trechos, 47 continham espículas e 52 continham apenas atividade não-epileptogênica normal. As amplitudes que extrapolassem a faixa considerada para espículas (até 200 $\mu \mathrm{V} \pm 10 \%$ ) foram cortadas e os valores foram substituídos pela média em cada trecho, para não gerar descontinuidades nos sinais. Isto foi feito para diminuir a influência de ruídos de alta freqüência e de piscadas, os quais freqüentemente apresentam amplitudes espúrias da ordem de 300 a $500 \mu \mathrm{V}$, que podem se tornar dominantes na análise.

Em seguida, os vetores de sinais foram organizados aleatoriamente na matriz de treinamento e os valores-alvo de cada vetor foram atribuídos. Para evitar-se que a rede treinada se tornasse sensível à posição da espícula no trecho de sinal, foi calculada a distribuição de amplitudes em 100 patamares, na faixa de $[-200,+200]$, para todos os vetores, formando um histograma de freqüências.

A rede $\mathrm{B}$ de reconhecimento de ondas agudas foi treinada com 180 trechos de $1 \mathrm{~s}$ de EEG, reconstruídos a partir do nível de decomposição 3, sendo que destes, 90 sinais continham ondas agudas e 90, apenas atividade normal. Foi necessário utilizar-se um conjunto de treinamento maior para a rede $\mathrm{B}$ em função da dificuldade de convergência encontrada inicialmente para um conjunto menor. As amplitudes espúrias também foram eliminadas e o histograma de freqüências foi calculado para cada vetor de treinamento.

As redes foram treinadas até que o erro quadrático médio fosse inferior a 0,05 ou até completarem-se 300.000 ciclos (Figura 6). A taxa de aprendizagem iniciou-se com valor 0,01 e sofreu incrementos de 1,05 e decrementos de 0,7 , a cada ajuste. $\mathrm{O}$ fator de momentum foi definido como 0,95 . 
Ao final do treinamento, os valores dos pesos e bias foram salvos em arquivos.

O resultado final da detecção foi arbitrado como sendo a soma lógica simples de ambas as entradas. Ou seja, se uma das duas redes detectasse um evento epileptogênico, a detecção seria considerada positiva. Se ambas detectassem um evento, o maior valor de saída seria o considerado para a detecção.
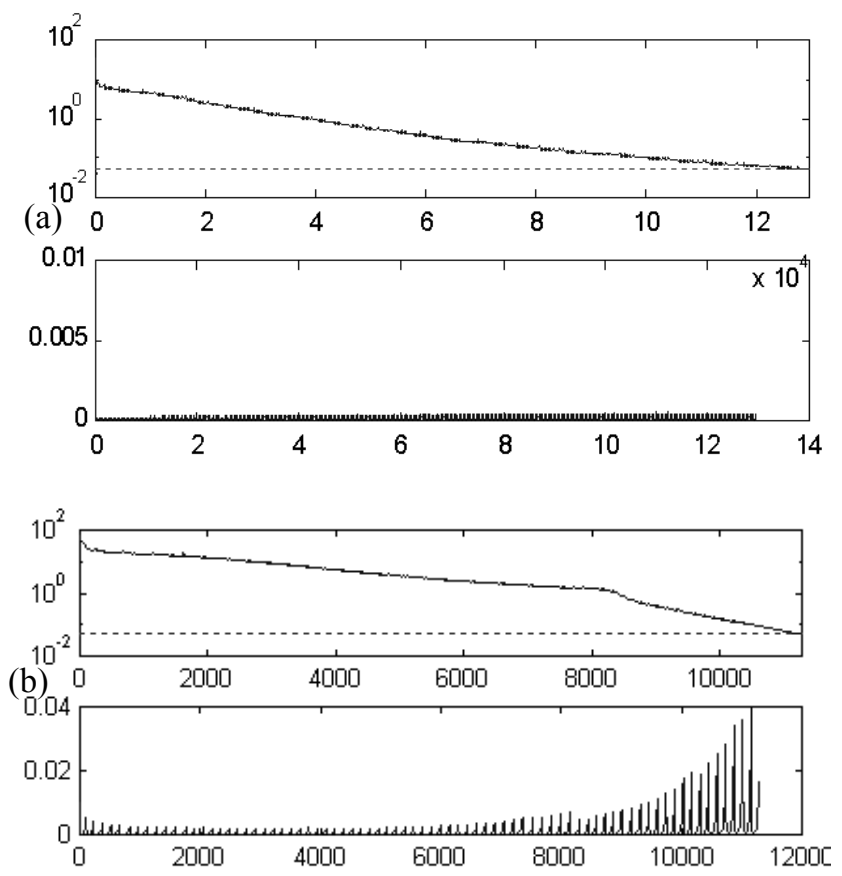

Figura 6 - Curvas de treinamento (Erro médio quadrático $\times$ número de ciclos) e variação da taxa de aprendizagem por número de ciclos: (a) para a rede neural de reconhecimento de espículas, e (b) para a rede de reconhecimento de ondas agudas.

\section{TESTES E RESULTADOS}

Para os testes com o sistema foram selecionados aleatoriamente 27 arquivos (3 de cada registro de paciente), com 15 segundos de duração cada um e com representação de padrões comportamentais e mentais distintos nos pacientes (durante o sono, vigília, estados de alerta, repouso, etc). Isto foi feito para garantir a variabilidade dos padrões de eventos contidos no conjunto de testes. Ou seja, foram testados 405 trechos de 100 pontos, totalizando 405 segundos de EEG.

Os limiares de detecção nas saídas de ambas as redes foram variados para se levantar as curvas de desempenho das mesmas. Os gráficos da figura 7 mostram os valores de sensibilidade e especificidade obtidos para cada uma das redes neurais.
Note-se que a escolha do limiar de detecção foi crítica no caso da rede $\mathrm{A}$, enquanto na rede $\mathrm{B}$ os valores de sensibilidade e especificidade mostraram-se praticamente estáveis, constantes, até o ponto de cruzamento.

Também é interessante notar que a rede $\mathrm{B}$ treinou muito mais rapidamente que a rede $\mathrm{A}$ (em aproximadamente 11.000 iterações, enquanto a rede para espículas demandou 130.000 iterações para convergir ao erro mínimo), o que sugere que um conjunto de treinamento maior $\mathrm{e}$ teoricamente mais variado pode facilitar a aprendizagem por parte da rede.
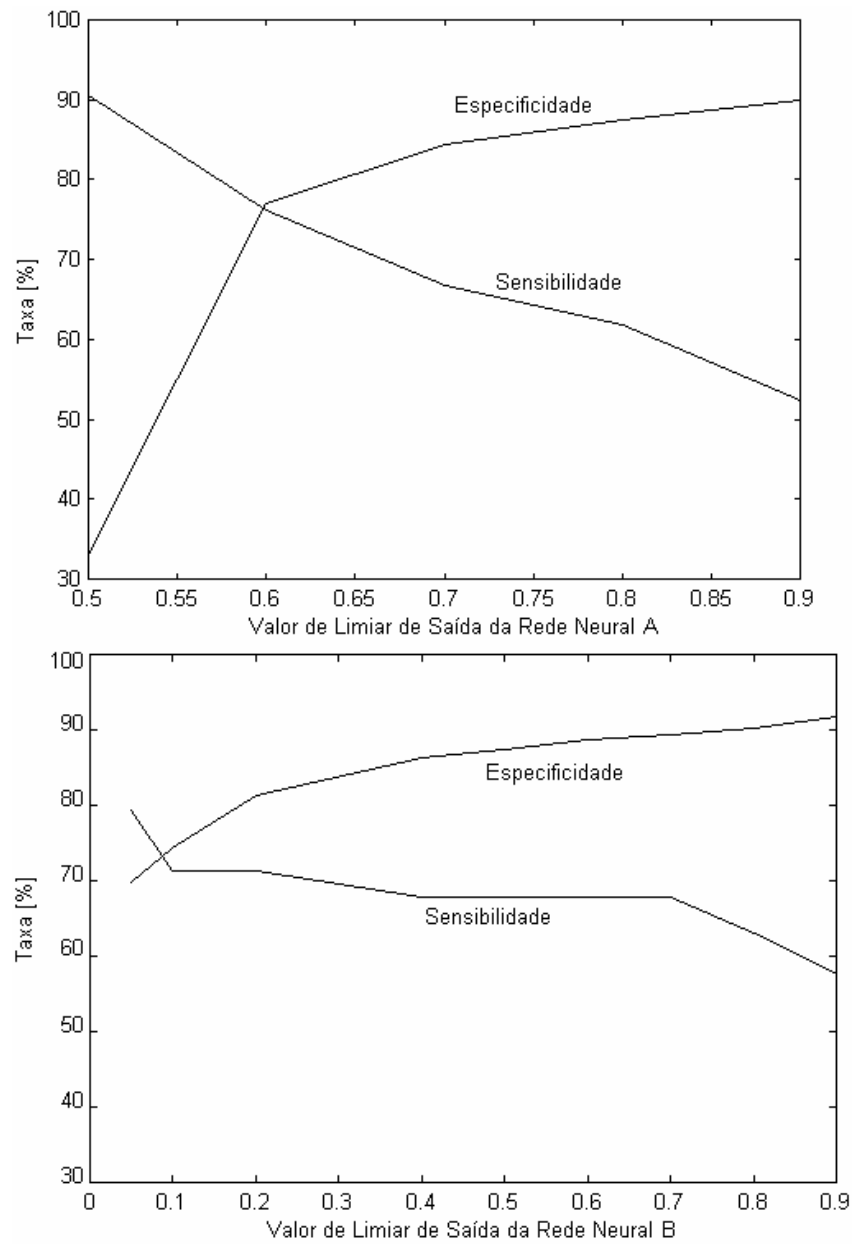

Figura 7 - Acima: curvas de desempenho da rede de reconhecimento de espículas, em função do limiar de saída da rede - cruzamento em 77\%, para um limiar de 0,6. Abaixo: curvas de desempenho da rede de reconhecimento de ondas agudas - cruzamento em $73 \%$, para um limiar de saída da rede de 0,1 .

Sobretudo, vale salientar que a organização deste banco de redes no sistema permite uma grande flexibilidade no ajuste do desempenho do mesmo. Ou seja, dependendo do sinal de EEG a ser analisado (alguns pacientes apresentam mais ondas agudas que espículas, e vice-versa), o limiar de uma 
das redes pode ser ajustado para que se obtenha mais sensibilidade que especificidade, enquanto o limiar da outra é ajustado para o contrário, por exemplo.

Ou ainda pode-se optar pelo uso dos pontos de cruzamento de ambas as redes, o que leva ao gráfico de desempenho da figura 8. Neste caso, a detecção foi considerada positiva quando a saída da rede $\mathrm{A}$ foi maior que 0,6 ou quando a saída da rede $\mathrm{B}$ foi maior que 0,1 e tomando-se o maior valor absoluto, em caso de dupla detecção.

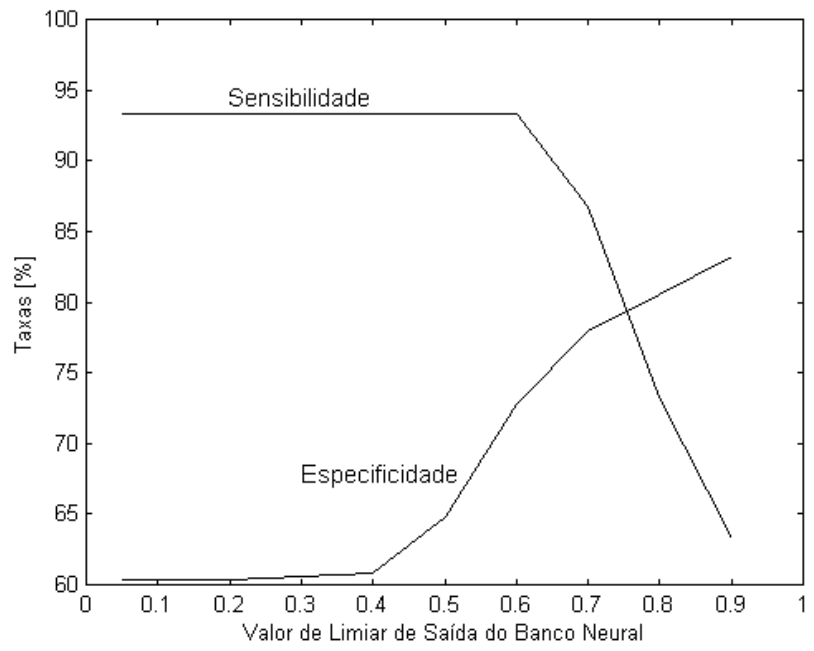

Figura 8 - Curvas de desempenho para o banco de redes neurais especializadas. O cruzamento ocorreu em 79,3\%, para um limiar de saída de 0,75 .

\section{CONCLUSÕES}

Os sistemas de reconhecimento automático de eventos epileptogênicos reportados por Wilson e Emerson (2002) não realizaram análises distintas para espículas e ondas agudas, tal como feito neste trabalho, apesar das diferenças morfológicas entre ambos os padrões. E normalmente os conjuntos de treinamento e teste utilizados contêm apenas um dos dois tipos de eventos (as espículas), apesar de ambos serem fortemente indicativos de epilepsia e freqüentes em sinais de pacientes epilépticos. Além disto, as maiores fontes de falso-positivos em detecção automática são, justamente, os eventos meramente epileptiformes morfologicamente mais semelhantes às ondas agudas, como as piscadas. Ou seja, quando um sistema automático é desenvolvido sem levar em conta a morfologia especial do padrão de ondas agudas, ou não será capaz de detectar estes eventos em especial, ou tenderá a confundi-los com padrões epileptiformes, gerando grande número de falso-positivos.

Quanto à eficiência do sistema, é importante notar-se também que o desempenho geral do banco de redes especializadas foi superior ao desempenho individual, principalmente no que se refere à sensibilidade. Das 30 espículas existentes no conjunto de teste, apenas 2 de um mesmo arquivo não foram detectadas*. Isto pode ser atribuído ao fato de que uma rede detectou eventos epileptogênicos que a outra não foi capaz de identifícar.

Os sinais que continham ruído de alta freqüência foram os responsáveis pela diminuição da taxa de especificidade, principalmente na rede A, que utiliza o menor nível de decomposição (o qual é inversamente proporcional à freqüência). Um algoritmo de identificação ou correção do ruído de alta freqüência poderia, em tese, elevar consideravelmente as taxas de especificidade do sistema. Outra alternativa seria, como já mencionado, ajustar-se os limiares de saída das redes de forma a aumentar a taxa de especificidade total, para o banco de redes. Assim, ajustando-se o limiar de saída da rede A para um valor superior a 0,7 , por exemplo, garantir-se-ia que esta seria menos sensível a quaisquer eventos, que não as espículas, incluindo-se os ruídos de alta freqüência.

Outro ponto a ser investigado é o aumento do tamanho do conjunto de treinamento da rede A, para verificar-se se desta forma o aprendizado desta rede se tornaria mais eficiente.

Enfim, vale salientar que o desempenho real obtido pelo sistema de quase $80 \%$ é compatível com os melhores trabalhos já desenvolvidos (Wilson e Emerson, 2002) e com a vantagem de se poder ajustar as taxas de desempenho, segundo as características particulares de cada sinal.

\section{AGRADECIMENTOS}

À CAPES, por propiciar a continuidade deste projeto, através do programa PRODOC.

\section{REFERÊNCIAS}

Argoud, F. I .M. (2001). Contribuição à Automatização da Detecção e Análise de Eventos Epileptiformes em Eletroencefalograma. Tese de Doutoramento, Instituto de Engenharia Biomédica, Universidade Federal de Santa Catarina.

Argoud, F. I. M.; F. M. Azevedo \& J. Marino Neto (2003). Bases Wavelet e sua Aplicação em Detecção Automática de Espículas em Eletroencefalograma. Submetido à Revista Brasileira de Engenharia Biomédica.

Attelis, C. E.; S. I. Isaacson \& R. O. Sirne (1997). Detection of epileptic events in

\footnotetext{
* Ambas as espículas estavam localizadas próximas aos limites dos trechos de $1 \mathrm{~s}$ e, por conseqüência, tiveram suas ondas lentas cortadas, o que poderia explicar o não-reconhecimento por parte do sistema.
} 
electroencephalograms using wavelet analysis. Annals of Biomedical Engineering, Vol. 25, no. 1, pp. 286-293.

De Azevedo, F. M. (1997). Uma proposta de modelos formais de neurônios e redes neurais artificiais. Anais do $3^{\circ}$. Congresso Brasileiro de Redes Neurais, Florianópolis, SC, pp. 503-514.

Dümpelmann, M. \& C.E. Elger (1999). Visual and Automatic Investigation of Epileptiform Spikes in Intracranial EEG Recordings. Epilepsia, Vol. 40, no. 3, pp.275-285.

Feucht, M.; K. Hoffmann; K. Steinberger; H. Witte; F. Benninger; M. Arnold; A. Doering (1997). Simultaneous spike detection and topographic classification in pediatric surface EEGs. NeuroReport. Vol. 8, pp. 2193-2197.

Gabor, A. J. \& M. Seyal (1992). Automated interictal EEG spike detection using artificial networks. Electroenceph. clin. Neurophysiol. Vol. 83, no. 1, pp. 271-280.

Gotman, J. \& P. Gloor (1976). Automatic recognition and quantification of interictal epileptic activity in the human scalp EEG. Electroenceph. clin. Neurophysiol., Vol. 41, pp. 513-529.

Gotman, J. \& L. Y. Wang (1991). State-dependent spike detection: concepts and preliminary results. Electroenceph. clin. Neurophysiol., Vol. 79, pp. 1119.

Hoffmann, K. et alii (1996). Analysis and classification of interictal spikes discharges in Benign Partial Epilepsy of Childhood on the basis of the Hilbert transform. Neuroscience Letters. Vol. 211, no. 1, pp. 195-198.

Kalayci, T. \& O. Özdamar (1995). Wavelet preprocessing for automated neural network detection of EEG spikes. IEEE Engineering in Medicine and Biology Society, pp. 160-166.

Khan, Y. U. \& J. Gotman (2003). Wavelet based automatic seizure detection in intracerebral electroencephalogram, Clin Neurophysiol, Vol. 114, pp. 898-908.

Pradhan, N.; P. K. Sadasivan \& G. R. Arunodaya (1996). Detection of seizure activity in EEG by an artificial neural network: a preliminary study. Comput. Biomed. Res., Vol. 29, no. 4, pp. 303-313.

Pereira, M. C. V. (2003). Tratamento de Sinais Bioelétricos para Processamento por Redes Neurais Artificiais.
Tese de Doutoramento, Instituto de Engenharia Biomédica, Universidade Federal de Santa Catarina, Florianópolis.

Schiff, S. J.; A. Aldoubri; M. Unser, S. Satao (1994). Fast wavelet transformation of EEG, Electroencephalogr Clin Neurophysiol, Vol. 91, pp. 442-455.

Sheng, Y. (1996 ). Wavelet Transform, in: The Transforms and Applications Handbook, Ed: A.D. Poularikas, 1 'edição, Boca Raton, Flórida: CRC Press and IEEE Press, pp. 747-827.

Stelle, A. L. \& R. A. Comley (1989). Portable analyzer for real-time detection of the epileptic pre-cursor. $R B E$. Vol. 6, no. 2, pp. 101-107.

Stelle, A. L. \& R. A. Comley (1990). The application of the Wigner distribution to the analysis of EEG signals. RBE. Vol. 7, no. 1, pp. 670-676.

Sweldens, W. (1996). The lifting scheme: A custom-design construction of biorthogonal wavelets. Appl. Comput. Harmon. Anal., Vol. 3, no. 2, pp. 186-200.

Webber, W. R.; B. Litt; K. Wilson; R. P. Lesser (1994). Practical detection of epileptiform discharges (EDs) in the EEG using an artificial neural network: a comparison of raw and parameterized EEG data. Electroenceph. clin. Neurophysiol., Vol. 91, pp. 194204.

Wilson, S. B.; C. A. Turner; R. G. Emerson; M. L. Scheuer (1999). Spike detection. Clin. Neurophysiol., Vol. 110, pp. 404-411.

Wilson, S. B. \& R. Emerson (2002). Spike Detection: a review and comparison of algorithms. Clin. Neurophysiol., Vol. 113, pp. 1873-1881. 